\title{
Scheduling of unit-length jobs with bipartite incompatibility graphs on four uniform machines
}

\author{
H. FURMANCZYK ${ }^{1 *}$ and M. KUBALE ${ }^{2}$ \\ ${ }^{1}$ Institute of Informatics, University of Gdańsk, Wita Stwosza 57, 80-309 Gdańsk, Poland \\ ${ }^{2}$ Department of Algorithms and System Modelling, Gdańsk University of Technology, Narutowicza 11/12, 80-233 Gdańsk, Poland
}

\begin{abstract}
In the paper we consider the problem of scheduling $n$ identical jobs on 4 uniform machines with speeds $s_{1} \geq s_{2} \geq s_{3} \geq s_{4}$, respectively. Our aim is to find a schedule with a minimum possible length. We assume that jobs are subject to some kind of mutual exclusion constraints modeled by a bipartite incompatibility graph of degree $\Delta$, where two incompatible jobs cannot be processed on the same machine. We show that the general problem is NP-hard even if $s_{1}=s_{2}=s_{3}$. If, however, $\Delta \leq 4$ and $s_{1} \geq 12 s_{2}, s_{2}=s_{3}=s_{4}$, then the problem can be solved to optimality in time $O\left(n^{1.5}\right)$. The same algorithm returns a solution of value at most 2 times optimal provided that $s_{1} \geq 2 s_{2}$. Finally, we study the case $s_{1} \geq s_{2} \geq s_{3}=s_{4}$ and give a 32/15-approximation algorithm running also in $O\left(n^{1.5}\right)$ time.
\end{abstract}

Key words: equitable coloring, NP-hardness, polynomial algorithm, scheduling, uniform machine.

\section{Introduction}

Let us imagine we have to arrange a dinner for 40 people and we have at our disposal 4 round tables with different numbers of seats (not greater than 16). We know that among our guests there are vegetarians and non-vegetarians. Moreover, each vegetarian is not on good terms with at most 4 non-vegetarians and vice-versa. Our task is to assign the people to the tables in such a way that no two persons who are not on good terms with one another seat at the same table. In the paper we show how to solve this and related problems.

Our problem can be expressed as the following scheduling problem. Suppose we have $n$ identical jobs $j_{1}, \ldots, j_{n}$, so we assume that they all have unit execution times, in symbols $p_{i}=1$, to be processed on four non-identical machines $M_{1}, M_{2}, M_{3}$, and $M_{4}$. These machines run at different speeds $s_{1} \geq s_{2} \geq s_{3} \geq s_{4}$, respectively. However, they are uniform in the sense that if a job is executed on a machine $M_{i}$, it takes $1 / s_{i}$ time units to be completed. It refers to the situation where the machines are of different generations, e.g. old and slow, new and fast, etc.

Our scheduling problem would be trivial if all the jobs were compatible. Therefore we assume that some pairs of jobs cannot be processed on the same machine. For example, this situation appears while processing groups of elements on non-identical serial batch machines, if the elements have various shapes that prevent some of them from being processed on the same machine. Such manufacturing systems are encountered as heat treatment operations in metalworking industries, as metal painted together or material transported together, etc. More precisely, we assume that each job is in conflict with at least 1 and at most 4 other jobs. Moreover, we assume that the underlying incompatibility graph $G$, whose vertices are jobs and

*e-mail: hanna@inf.ug.edu.pl edges correspond to pairs of jobs being in conflict, is a bipartite graph (without isolated vertices). For example, all graphs in our figures are bipartite. Notice that two jobs being in conflict may be executed in intersecting time intervals. A load of $k$ jobs on $M_{i}$ machine requires the processing time $k / s_{i}$, and all the jobs are ready for processing at the same time. Alternatively, if a load on $M_{i}$ is not given explicitly, we are using the notation $C\left(M_{i}\right)$ to mean the schedule length on the machine $M_{i}$. By definition, each load forms an independent set (color) in $G$. Therefore, we will be using the terms job/vertex and load/color/independent set interchangeably. Since all the tasks have to be executed, the problem is to find a 4-coloring, i.e. a decomposition of $G$ into 4 independent sets $I_{1}, I_{2}, I_{3}$, and $I_{4}$ such that the schedule length $C_{\max }=\max \left\{\left|I_{i}\right| / s_{i}: i=1, \ldots, 4\right\}$ is minimized, in symbols $Q 4 \mid p_{i}=1, G=$ bipartite $\mid C_{\max }$.

There are several papers devoted to chromatic scheduling in the presence of mutual exclusion constraints. Boudhar in $[1,2]$ studied the problem of batch scheduling with complements of bipartite and split graphs, respectively. Finke et al. [3] considered this problem with complements of interval graphs. Other models of batch scheduling with incompatibility constraints were studied in $[4,5]$. In all the papers the authors assumed identical parallel machines. However, to the best of our knowledge little work has been done on scheduling problems with uniform machines involved (cf. $[6,7])$.

The rest of this paper is organized as follows. In Section 2 we show that the general problem is NP-hard even if $s_{1}=s_{2}=s_{3}$. In Section 3 we show that if $s_{1} \geq 12 s_{2}, s_{2}=s_{3}=s_{4}$, then the problem can be solved to optimality in time $O\left(n^{1.5}\right)$ provided that the degree of $G$ is $\Delta \leq 4$. The same algorithm returns a solution of value at most 2 times optimal provided that $s_{1} \geq 2 s_{2}$, $s_{2}=s_{3}=s_{4}$. In Section 4 we study the case $s_{1} \geq s_{2} \geq s_{3}=s_{4}$ and give an $O\left(n^{1.5}\right)$-time $32 / 15$-approximation algorithm in all such cases. Finally, we discuss possible extensions of our model to more than four machines. 


\section{NP-completeness proof}

We begin with introducing a few basic notions concerning graph coloring. Given graph $G=(V, E)$, a $k$-coloring of $G$ is a mapping $c: V \rightarrow\{1, \ldots, k\}$ such that for all edges $\{u, v\} \in E$ we have $c(u) \neq c(v)$. The smallest $k$ for which $G$ is $k$-colorable is called the chromatic number of $G$ and denoted $\chi(G)$. A graph $G=(V, E)$ is said to be equitably $k$-colorable if and only if its vertex set can be partitioned into independent sets $V_{1}, \ldots, V_{k} \subset V$, possibly empty, such that $\| V_{i}|-| V_{j} \mid \leq 1$ for all $i, j=1, \ldots, k$. The smallest $k$ for which $G$ admits such a coloring is called the equitable chromatic number of $G$ and denoted $\chi_{=}(G)$. Graph $G$ has a semi-equitable $k$-coloring $(k \geq 3)$, if there exists a partition of its vertices into independent sets $V_{1}, \ldots, V_{k} \subset V$ such that one of these subsets, say $V_{i}$, is of size $\notin\{\lfloor n / k\rfloor,\lceil n / k\rceil\}$, and the remaining subgraph $G-V_{1}$ is equitably $\$(\mathrm{k}-1) \$$-colorable. In the following we will say that graph $G$ has $(k-1)$-coloring to express explicitly a partition of $V$ into $k$ independent sets. If, however, only the cardinalities of color classes are important, we will use the notation $\left[\left|V_{1}\right|, \ldots,\left|V_{k}\right|\right]$. For example, the graph in Fig. 1 has one equitable coloring of type $[3,2,2,2]$, one semi-equitable coloring of type $[6,1,1,1]$ and several other types of colorings.

Let us recall some basic facts concerning the colorability of bipartite graphs. First of all, for any bipartite graph $G$ we have $\chi(G)=2$. Such a 2-coloring can be obtained in time proportional to the size of $G$ while traversing it in a DFS order. Moreover, Chen and Yen [8] proved that any bipartite graph $G$ with $\Delta \geq 2$ is equitably $\Delta$-colorable in linear time if and only if $G$ is different from a complete bipartite graph $K_{2 q+1,2 q+1}$ for all $q \geq 1$.

The maximal size of an independent set in $G$ is called the independence number of $G$ and denoted $\alpha(G)$. Since $\alpha(G) \chi(G) \geq n$,

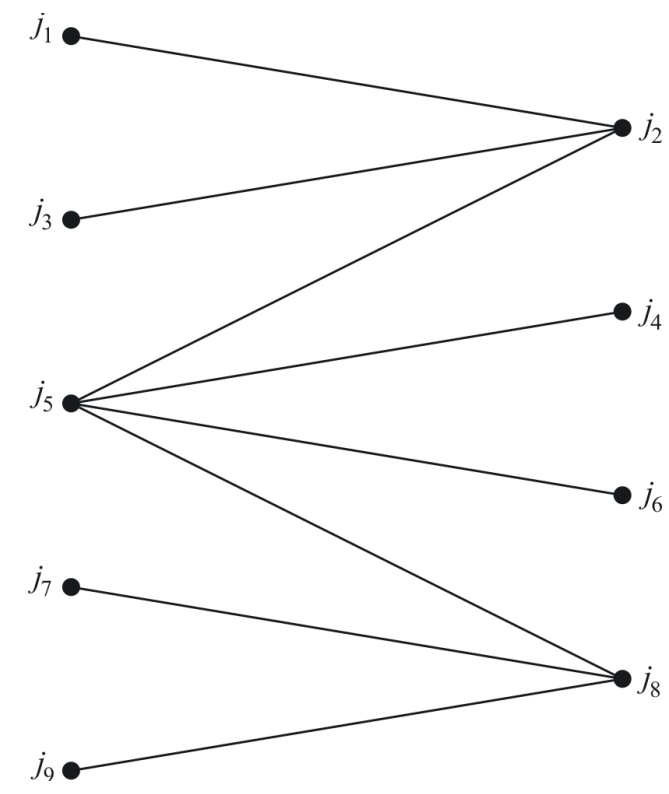

Fig. 1. Example of incompatibility graph we have a lower bound $\alpha(G) \geq n / \chi(G)$ on it. On the other hand, the maximal gap between the sizes of independent sets in a bipartite graph is for $K_{1, \Delta}$. This follows that $\alpha(G) \leq n \Delta /(\Delta+1)$. Since in our case $\chi(G)=2$ and $\Delta \leq 4$, we have

$$
n / 2 \leq \alpha(G) \leq 4 n / 5
$$

Note that an independent set of size $\alpha(G)$ can be computed in $O\left(n^{1.5}\right)$ time by finding a maximum matching in $G$ (see Hopcroft and Karp [9]), since one of the two endpoints of each edge in the maximum matching belongs to the complement of maximum independent set in $G$.

In the following we will need the partition into bounded independent sets (PIBIS) problem, which is defined as follows: Given a graph $G=(V, E)$ and positive integers $k, l$, the question is whether there is a partition of $V$ into independent sets $V_{1}, \ldots, V_{k} \subset V$ such that $\left|V_{i}\right| \leq l$ for each $i=1, \ldots, k$. We shall call this the $\operatorname{PIBIS}(G, k, l)$ problem. Since $\operatorname{PIBIS}(G, k, n)$ is a well-known NP-complete $k$-coloring problem, so is $\operatorname{PIBIS}(G, k, l), l<n$. Bodlaender and Jansen [10] proved that the $\operatorname{PIBIS}(G, 3, l)$ problem remains NP-complete even if $G$ is bipartite. Now we are ready to prove Theorem 2.1.

Theorem 2.1. The $Q 4 \mid p_{i}=1, G=$ bipartite $\mid C_{\max }$ problem is $N P$-hard even if $s_{1}=s_{2}=s_{3}$.

Proof. We prove by reduction from the $\operatorname{PIBIS}(G, 3, l)$ problem. Suppose we have an instance of $\operatorname{PIBIS}(G, 3, l)$, i.e. we have a bipartite graph $G$ and we want to know whether there exists a partition of its vertices into three independent sets, each of size $\leq l$. We construct the following instance of a scheduling decision problem: machine speeds for $M_{1}, M_{2}$, and $M_{3}$ are $s_{1}=s_{2}=s_{3}=1$. Machine $M_{4}$ is of speed $s_{4} \ll 1 / n$ and the limit on schedule length is $l$. The question is whether there exists a schedule of length at most $l$. The membership of this problem in class NP is obvious.

The existence of a schedule of length $\leq l$ implies the existence of a 3-partition of $G$ into independent sets of size at most $l$, since no job can be allocated to $M_{4}$.

If $G$ has a 3-coloring with at most $l$ vertices in each color then our scheduling problem has clearly a solution of length at most $l$, since each color class can be regarded as a load on some $M_{i}, i \leq 3$.

The NP-hardness of $Q 4 \mid p_{i}=1, G=$ bipartite $\mid C_{\max }$ follows from the fact that its decision version is NP-complete.

\section{Algorithm for the case $s_{2}=s_{3}=s_{4}$}

Since our scheduling problem is NP-hard, we have to propose an approximation algorithm for it. First of all notice that if all the machines are identical then the scheduling problem becomes trivial since any equitable 4-coloring of $G$ solves the problem to optimality. Therefore we assume herein that $s_{1} \gg s_{2}=s_{3}=s_{4}$ and the incompatibility graph $G$ is of degree at most 4 .

In the following we will need a lower bound on the schedule length which we call an ideal schedule. Let $s=s_{1}+s_{2}+s_{3}+s_{4}$. A schedule in which all the machines finish at the same time 
(a)

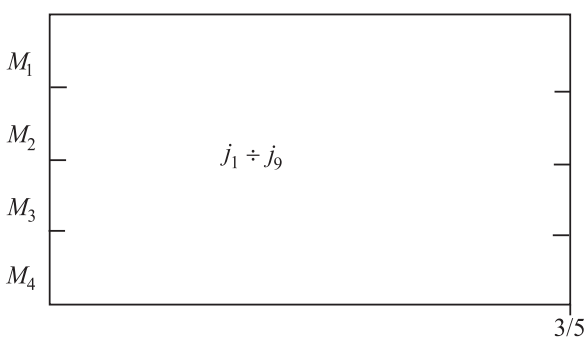

(b)

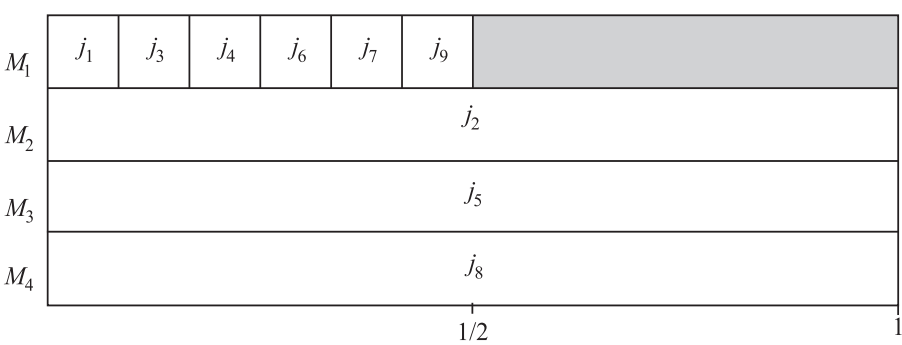

Fig. 2. Gantt chart of a schedule for graph of Fig. 1$\}$ when $s_{1}=12$, $s_{2}=s_{3}=s_{4}=1$ : (a) ideal; (b) optimal

is said to be ideal. Note that the length of the ideal schedule is $n / s$. Since the number of jobs on each machine must be an integer, the ideal schedule need not be optimal. Such a situation is illustrated in Fig. 2.

The general idea behind our heuristics is to find a semi-equitable coloring in which the largest possible independent set in $G$ is allocated to machine $M_{1}$ and the remaining job vertices are spread equitably within machines $M_{2}, M_{3}$, and $M_{4}$. This leads to the following Algorithm 1 for optimal/suboptimal scheduling in this case.

The most time-consuming Step 1 of Algorithm 1 can be done in $O\left(n^{1.5}\right)$ time [9].

Theorem 3.1. If $s_{1} \geq 12 s_{2}$ and $s_{2}=s_{3}=s_{4}$ then Algorithm 1 returns an optimal solution.

Algorithm 1. Scheduling in case $s_{2}=s_{3}=s_{4}$

Input: $n$-vertex graph $G$ with $\Delta(G) \leq 4$ and machine speeds $s_{1} \gg s_{2}=s_{3}=s_{4}$.

Output: Optimal/suboptimal schedule.

1. Find a maximum independent set $I_{1}$ in $G$.

2. If exactly one of components of $G-I_{1}$ is isomorphic to $K_{3,3}$, then set $I_{1}=I_{1} \cup\{v\}-\{u\}$ as shown in Fig. 3.

3. Assign $M_{1} \leftarrow I_{1}$.

4. Find an equitable $(A, B, C)$-coloring of $G-I_{1}$ according to [8].

5. Assign $M_{2} \leftarrow A, M_{3} \leftarrow B, M_{4} \leftarrow C$. (a)

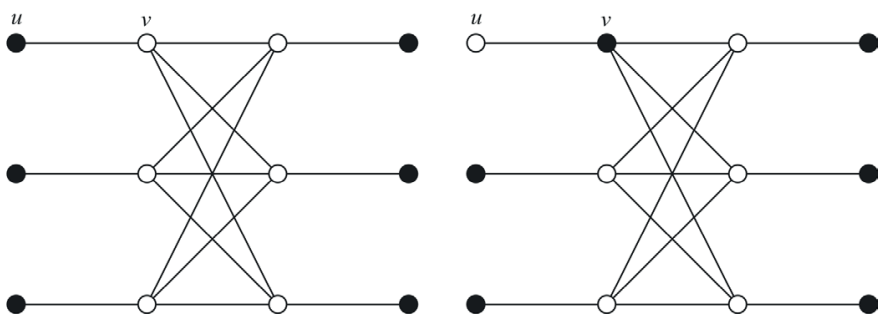

Fig. 3. $K_{3,3}$ and its neighborhood (vertices in black belong to $I_{1}$ ): (a) before interchange; (b) after interchange

Proof. Let $G$ be an $n$-vertex incompatibility graph $G$ of degree $\Delta \leq 4$ and let $I_{1}$ be a maximum cardinality independent set in $G$.

First notice that if exactly one of the components of $G-I_{1}$ is isomorphic to $K_{3,3}$ then $I_{1}$ must contain 6 vertices from the neighborhood of $K_{3,3}$, since otherwise $I_{1}$ would not be the maximum independent set. Therefore, we have to interchange one of them, say $u$, with its neighbor $v$ belonging to $K_{3,3}$, i.e. set $I_{1}:=I_{1} \cup\{v\}-\{u\}$.

Now, we will show that $G-I_{1}$ is of degree at most 3. If $\Delta(G) \leq 3$, there is nothing to prove. So suppose that $\Delta(G)=4$ and let $v$ be any vertex of degree 4 . If each such $v \in I_{1}$ then $\Delta\left(G-I_{1}\right) \leq 3$. If $v \notin I_{1}$ then at least one of its neighbors, say $u$, belongs to $I_{1}$ since otherwise edge $\{u, v\}$ would not be covered by a minimal vertex cover and, due to König's theorem [11], $I_{1}$ would not be maximal.

Let $C\left(M_{i}\right.$ be the schedule length on machine $M_{i}, i=1, \ldots, 4$. Without loss of generality we may assume that $s_{1}=12 s_{2}$. Then the ideal schedule is of length $n / s=\frac{1}{15} n / s_{2}$. By inequality (1) it follows that $\left|I_{1}\right| \leq \frac{4}{5} n$. Thus $C\left(M_{1}\right) \leq \frac{4}{5} n /\left(12 s_{2}\right)$, which is equal to the ideal schedule length. Since $G-I_{1}$ is a collection of subcubic bipartite graphs different from $K_{3,3}$, we can find an optimal scheduling on $M_{2}, M_{3}$, and $M_{4}$ by equitable 3 -coloring of $G-I_{1}$. In this way the remaining jobs are spread evenly among the three machines $M_{i}, i \geq 2$ which gives $\max \left\{C\left(M_{i}\right): i=2,3,4\right\}=C_{\max }^{*}$, because one cannot do better by moving a job from $M_{i}$ to $M_{1}$ as $I_{1}$ is maximal. This completes the proof of Theorem 3.1.

Corollary 3.2. If $s_{1} \geq 2 s_{2}$ and $s_{2}=s_{3}=s_{4}$ then Algorithm 1 returns a solution of value at most 2 times $C_{\max }^{*}$.

Proof. Without loss of generality we may assume that $s_{1}=2 s_{2}$. In this case the ideal schedule length is $n / s=n /\left(5 s_{2}\right)=\frac{2}{5} n / s_{1}$. Let us consider two extremal cases given in inequality (1).

Case 1: $\left|I_{1}\right|=\lceil n / 2\rceil$.

Algorithm 1 returns a solution on $M_{1}$ of length $C\left(M_{1}\right)=$ $=\lceil n / 2\rceil / s_{1}$ which is less than $\frac{4}{5} n / s_{1}$, i.e. twice the ideal schedule length. The remaining jobs are spread evenly among three machines $M_{i}, i \geq 2$, which gives $C\left(M_{1}\right) \cong\lfloor n / 2\rfloor$ $/\left(s_{2}+s_{3}+s_{4}\right)=\lfloor n / 2\rfloor /\left(1.5 s_{1}\right)=\frac{2}{3}\lfloor n / 2\rfloor / s_{1}$ which is less than $\frac{4}{5} n / s_{1}$, i.e. twice the ideal schedule length. The thesis holds in Case 1. 
Case 2: $\left|I_{1}\right|=\lfloor 4 n / 5\rfloor$.

Then $C\left(M_{1}\right)=\lfloor 4 n / 5\rfloor / s_{1}$ which is less than or equal to $\frac{4}{5} n / s_{1}$, i.e. twice the ideal schedule length. The remaining jobs are spread evenly among $M_{i}, i \geq 2$, which gives $C\left(M_{i}\right) \cong\lceil n / 5\rceil /$ $\left(3 s_{2}\right)=\lceil n / 5\rceil /\left(1.5 s_{1}\right)=\frac{2}{3}\lceil n / 5\rceil / s_{1}<\frac{4}{5} n / s_{1}$, i.e. twice the ideal schedule length. The thesis holds in Case 2.

The reader can check that the thesis holds in the remaining cases as well.

The worst-case instance for Algorithm 1 when $s_{1}=2 s_{2}$ and $G=3 K_{1,4}$ is shown in Fig. 5 .
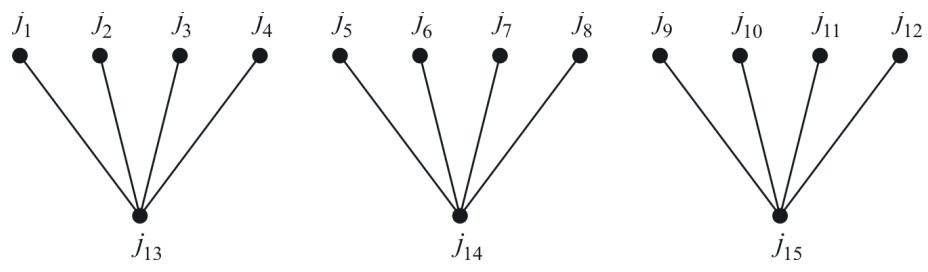

Fig. 4. Example of incompatibility graph $G=3 K_{1,4}$

(a)

\begin{tabular}{|c|c|c|c|c|c|c|c|c|c|c|c|c|}
\hline$M_{1}$ & $j_{1}$ & $j_{2}$ & $j_{3}$ & $j_{4}$ & $j_{5}$ & $j_{6}$ & $j_{7}$ & $j_{8}$ & $j_{9}$ & $j_{10}$ & $j_{11}$ & $j_{12}$ \\
\hline$M_{2}$ & \multicolumn{12}{|c|}{$j_{13}$} \\
\hline$A_{3}$ & \multicolumn{12}{|c|}{$j_{14}$} \\
\hline$M_{4}$ & \multicolumn{12}{|c|}{$j_{15}$} \\
\hline & & & & & & & & & & & & \\
\hline
\end{tabular}

(b)

\begin{tabular}{|c|c|c|c|c|}
\hline$M_{1}$ & $j_{1}$ & $j_{3}$ & $j_{5}$ & $j_{6}$ \\
\hline$M_{2}$ & $j_{7}$ & $j_{8}$ & & \\
\hline & $j_{10}$ & $j_{11}$ & & $i_{12}$ \\
\hline & $j_{13}$ & $j_{14}$ & & $j_{15}$ \\
\hline
\end{tabular}

Fig. 5. Gantt chart of a schedule for Algorithm 1 with graph from Fig. 4 when $s_{1}=2 s_{i}, 2 \leq i \leq 4$ (a) worst-case schedule; (b) optimal schedule

\section{Algorithms for the case $s_{3}=s_{4}$}

In this case we have two fast machines $M_{1}, M_{2}$, and two slow machines $M_{3}, M_{4}$. Therefore it is reasonable to apply a maximum independent set algorithm twice: first towards $G$, which results in a set $I_{1}$, and then towards $G-I_{1}$. This idea leads us to an approximation Algorithm 2.

\section{Algorithm 2. Scheduling in case $s_{3}=s_{4}$}

Input: $n$-vertex graph $G$ with $\Delta(G) \leq 4$ and machine speeds $s_{1} \geq s_{2} \geq s_{3}=s_{4}$.

Output: Suboptimal schedule.

1. Find $I_{1}$ a maximum independent set in $G$.

2. Find $I_{2}$ a maximum independent set in $G-I_{1}$.

3. Find an equitable $(C, D)$-coloring of $G-I_{1}-I_{2}$.

4. Assign $M_{1} \leftarrow I_{1}, M_{2} \leftarrow I_{2}, M_{3} \leftarrow C, M_{4} \leftarrow D$.

Lemma 4.1. If $s_{1} \geq s_{2} \geq 3 s_{3}=3 s_{4}$ then Algorithm 2 runs in time $O\left(n^{1.5}\right)$ to find a solution of value at most $32 / 15$ times $C_{\max }^{*}$

Proof. The complexity of Algorithm 2 is obvious since both Steps 1 and 2 can be done in time $O\left(n^{1.5}\right)$ [9] while Step 3 is linear.

Let us consider the accuracy of Algorithm 2. For this reason we may assume, without loss of generality, that $s_{1}=s_{2}=3 s_{3}$. In this case the ideal schedule length is $n /\left(8 s_{3}\right)$. Note that the subgraph $G-I_{1}$ is of degree at most 3 . This subgraph may be connected or disconnected. Its order is between $\lfloor n / 5\rfloor$ and $\lceil n / 2\rceil$. Since it is bipartite, we have $\lceil n / 10\rceil \leq \alpha\left(G-I_{1}\right) \leq\lfloor n / 2\rfloor$. Let $I_{2}$ be a maximum cardinality independent set in $G-I_{1}$. The subgraph $G-I_{1}-I_{2}$ can be equitably colored with 2 colors, since $\Delta\left(G-I_{1}-I_{2}\right) \leq 2$. In this way we can get a 4-coloring ranging from $[\lfloor 4 n / 5\rfloor,\lceil n / 5\rceil, 0,0]$ through $[\lceil n / 2\rceil,\lfloor n / 2\rfloor, 0,0]$ till $[\lceil n / 2\rceil,\lceil 3 n / 8\rceil,\lceil n / 16\rceil,\lfloor n / 16]]$. Therefore the schedule length is at most $\lfloor 4 n / 5\rfloor / s_{1}$. Thus

$$
\frac{\mathrm{Alg}_{2}}{C_{\max }^{*}} \leq \frac{\lfloor 4 n / 5\rfloor / s_{1}}{n /\left(8 s_{3}\right)} \leq \frac{4 n /\left(5 s_{1}\right)}{n /\left(8 s_{3}\right)}=\frac{4 / 15}{1 / 8}=\frac{32}{15}
$$

In contrast to Algorithm 1, which guarantees an optimal solution to our scheduling problem if $s_{1} \geq 12 s_{2}$, no such a guarantee exists for Algorithm 2. In other words, there is no bound on $s_{2} / s_{3}$ which guarantees that Algorithm 2 solves the problem to optimality. In fact, consider graph $G$ depicted in Fig. 6 and assume that $s_{1}=s_{2}$. Algorithm 2 when applied to $G$ finds a col- (a)

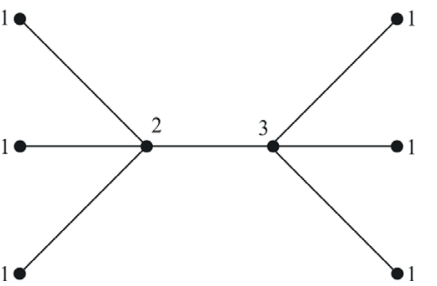

(b)

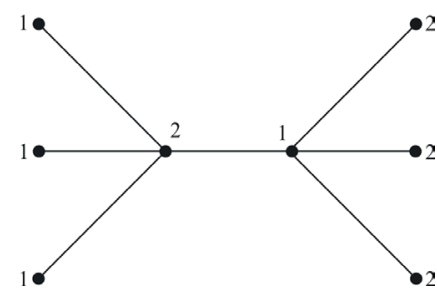

Fig. 6. Graph $G$ with two colorings: (a) of type $[6,1,1,0]$; (b) of type $[4,4,0,0]$ 
oring of type $[6,1,1,0]$, which leads to a schedule of length $\max \left\{6 / s_{1}, 1 / s_{3}\right\}$. A better coloring is $[4,4,0,0]$ which results in the schedule length of value $4 / s_{2}=4 / s_{1}<6 / s_{1}$, irrespective of $s_{3}$.

Now let us consider an approach based on equitable 4-coloring of $G$. The fact that every bipartite graph of degree $\leq 4$ is equitably 4-colorable was proved by Chen and Yen [8]. In this case we get a coloring of type $\lceil\lceil/ 4\rceil,\lceil(n-1) / 4\rceil,\lceil(n-2) / 4\rceil$, $\lceil(n-3) / 4\rceil]$. Hence the schedule length is determined by $C\left(M_{3}\right)=\lceil(n-2) / 4\rceil / s_{3}$. The algorithm of this kind is presented as Algorithm 3 below.

\section{Algorithm 3. Scheduling in case $s_{3}=s_{4}$}

Input: $n$-vertex graph $G$ with $\Delta(G) \leq 4$ and machine speeds $s_{1} \geq s_{2} \geq s_{3}=s_{4}$.

Output: Suboptimal schedule.

1. Find an equitable $(A, B, C, D)$-coloring of $G$ by applying a procedure described in [8]..

2. Order the independent sets so that $|A| \geq|B| \geq|C| \geq$ $\geq|A|-1$.

3. Assign $M_{1} \leftarrow A, M_{2} \leftarrow B, M_{3} \leftarrow C, M_{4} \leftarrow D$.

The worst-case ratio of Algorithm 3 is bounded above by

$$
\frac{C\left(M_{3}\right)}{C\left(M_{2}\right)}=\frac{\lceil(n-2) / 4\rceil / s_{3}}{\lceil(n-1) / 4\rceil / s_{2}} \cong \frac{s_{2}}{s_{3}}
$$

which can be arbitrarily large if $s_{3}$ is constant and $s_{2}$ tends to infinity. We have the following Lemma 4.2.

Lemma 4.2. If $s_{1} \geq s_{2}$ and $s_{2} \leq 3 s_{3}=3 s_{4}$ then Algorithm 3 runs in time $O(n)$ to find a solution of value at most $2 C_{\text {max }}^{*}$.

Proof. Without loss of generality we may assume that $s_{1}=s_{2}=$ $=3 s_{3}=3 s_{4}$. In this case the length of ideal schedule is $n /\left(8 s_{3}\right)$. In the following we consider two cases depending on the parity of $n$.

Case 1: $n=2 k$.

We have $C\left(M_{3}\right)=\lceil(n-2) / 4\rceil / s_{3}=\lceil(k-1) / 2\rceil / s_{3} \leq \frac{1}{2} k / s_{3}=$ $=2 n /\left(8 s_{3}\right) \leq 2 C_{\max }^{*}$.

Case 2: $n=8 k-1,8 k+3,8 k+5$.

In this case the ideal schedule is not optimal, since its length is not an integer. The example of the schedule for the case $n=8 k-1$ is shown in Fig. 7 .

If $n=8 k-1$ then an optimal solution corresponds to a coloring of $G$ of type $[3 k, 3 k, k, k-1]$. Hence $C\left(M_{3}\right)=$ $=\lceil(8 k-3) / 4\rceil / s_{3}=2 k / s_{3}=2 C_{\max }^{*}$. (a)

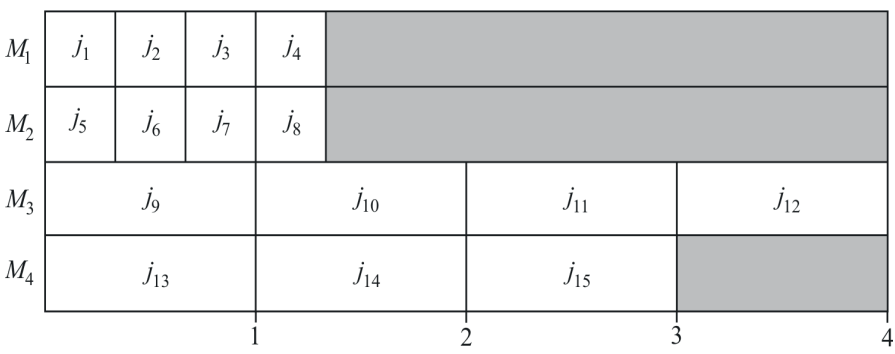

(b)

\begin{tabular}{|l|l|l|l|l|l|l|}
\hline$M_{1}$ & $j_{1}$ & $j_{2}$ & $j_{3}$ & $j_{4}$ & $j_{5}$ & $j_{6}$ \\
\cline { 2 - 6 }$M_{2}$ & $j_{7}$ & $j_{8}$ & $j_{9}$ & $j_{10}$ & $j_{11}$ & $j_{12}$ \\
\cline { 2 - 6 }$M_{3}$ & \multicolumn{3}{|c|}{$j_{13}$} & \multicolumn{3}{|c}{$j_{14}$} \\
\cline { 2 - 6 }$M_{4}$ & \multicolumn{3}{c}{1}
\end{tabular}

Fig. 7. Gantt chart of a schedule for Algorithm 3 with graph of Fig. 4 when $s_{1}=s_{2}=3 s_{3}=3 s_{4}$ : (a) worst-case schedule; (b) optimal schedule

If $n=8 k+1$ then an optimal solution corresponds to a coloring of $G$ of type $[3 k+1,3 k, k, k]$. That is why $C\left(M_{3}\right)=\lceil(8 k-1) / 4\rceil / s_{3}=2 k / s_{3}<2\left(k+\frac{1}{3}\right) / s_{3}=2 C_{\text {max }}^{*}$.

If $n=8 k+3$ then an optimal solution corresponds to a coloring of $G$ of type $[3 k+2,3 k+1, k, k]$. Thus $C\left(M_{3}\right)=$ $=\lceil(8 k+1) / 4\rceil / s_{3}=(2 k+1) / s_{3}<2\left(k+\frac{2}{3}\right) / s_{3}=2 C_{\max }^{*}$.

If $n=8 k+5$ then an optimal solution corresponds to a coloring of $G$ of type $[3 k+3,3 k+2, k, k]$. Therefore $C\left(M_{3}\right)=$ $=\lceil(8 k+3) / 4\rceil / s_{3}=(2 k+1) / s_{3}<2(k+1) / s_{3}=2 C_{\max }^{*}$.

The above considerations lead us to the following universal algorithm

Algorithm 4. Scheduling in case $s_{3}=s_{4}$

Input: $n$-vertex graph $G$ with $\Delta(G) \leq 4$ and machine speeds $s_{1} \geq s_{2} \geq s_{3}=s_{4}$.

Output: 2-approximate schedule.

1. If $s_{2}<3 s_{3}$ then call Algorithm 2 else call Algorithm 3.

Theorem 4.3. Algorithm 4 runs in time $O\left(n^{1.5}\right)$ to produce a solution of value at most $32 / 15$ times $C_{\max }^{*}$. 


\section{Final remarks}

Our results can be generalized to more than 4 machines. First, suppose that the number of machines $m>4$. Then the general problem $Q m \mid p_{i}=1, G=$ bipartite $\mid C_{\max }$ remains NP-hard if $s_{1}=s_{2}=s_{3}$. In fact, if $s_{4}=\cdots=s_{m} \ll 1 / m n$ then by the same argument as that used in the proof of Theorem 2.1 we get the desired result.

Secondly, the problem $Q m \mid p_{i}=1, G=$ bipartite $\mid C_{\max }$ can be solved to optimality in time $O\left(n^{1.5}\right)$ by using an algorithm similar to Algorithm 1, if $s_{1} \geq m(m-1) s_{2}, s_{2}=\cdots=s_{m}$ and $\Delta \leq m$. This is so because under these assumptions the ideal schedule length is $n /\left(s_{1}+s_{2}+\cdots+s_{m}\right) \geq n /\left(s_{1}+s_{1} / m\right)=$ $=n /\left(\left(1+m^{-1}\right) s_{1}\right)=n m /\left((m+1) s_{1}\right)$. On the other hand, since $\alpha(G) \leq n \Delta /(\Delta+1) \leq n m /\left((m+1) s_{1}\right)$ so $C\left(M_{1}\right) \leq n m /\left((m+1) s_{1}\right)$. It follows that the schedule length on $M_{1}$ is not longer than the ideal schedule length. Thus an optimal solution to the whole system is determined by the optimal schedule on machines $M_{2}, \ldots, M_{m}$, since we cannot do better by moving any job from $M_{i}$ to $M_{1}$ as the load on the first machine is maximal.

Thirdly, the above algorithm produces a schedule of length at most $m / 2$ times optimal, if $\frac{1}{2} s_{1} \geq s_{2}=\cdots=s_{m}$. This is so because $C\left(M_{1}\right) \leq n m /\left((m+1) s_{1}\right)$, while the ideal schedule length in this case is $n /\left(s_{1}+s_{2}+\cdots+s_{m}\right) \geq n /\left(s_{1}+\frac{1}{2}(m-1) s_{1}\right)=$ $=2 n /\left((m+1) s_{1}\right)$.

Another way of extending our model is allowing time window constraints imposed on the machines. In this direction of study the model of graph coloring with forbidden colors would be useful [12].

Acknowledgements. The project has been partially supported by Narodowe Centrum Nauki under contract DEC-2011/02/A/ ST6/00201.

\section{REFERENCES}

[1] M. Boudhar, "Scheduling a batch processing machine with bipartite compatibility graphs", Math. Methods Oper. Res. 57, 513-527 (2003).

[2] M. Boudhar, "Scheduling on a batch processing machine with split compatibility graphs", J. Math. Modell. Algorithms 4, 391- 407 (2005).

[3] G. Finke, V. Jost, M. Queyranne, A. Sebó, "Batch processing with interval graph compatibilities between tasks", Disc. Appl. Math. 156, 556-568 (2008).

[4] M. Demange, D. de Werra, J. Monnot, V.Th. Paschos, "Time slot scheduling of compatible jobs", J. Scheduling 10, 111-127 (2007).

[5] D. de Werra, M. Demange, J. Monnot, V.Th. Paschos, "A hypocoloring model for batch scheduling", Disc. Appl. Math. 146, 3-26 (2005).

[6] H. Furmańczyk, M. Kubale, "Scheduling of unit-length jobs with cubic incompatibility graphs on three uniform machines", Disc. Appl. Math., in print, available online 22 Feb. 2016.

[7] S.-S. Li, Y.-Z. Zhang, "Serial batch scheduling on uniform parallel machines to minimize total completion time", Inf. Process. Lett. 114, 692-695 (2014).

[8] B.-L. Chen, C.-H. Yen, "Equitable $\Delta$-coloring of graphs", Disc. Math. 312, 1512-1517 (2012).

[9] J.E. Hopcroft, R.M. Karp, "An $n^{5 / 2}$ algorithm for maximum matchings in bipartite graphs", SIAM J. Comput. 2, 225-231 (1973).

[10] H.L. Bodlaender, K. Jansen, "On the complexity of scheduling incompatible jobs with unit-times", LNCS 711, 291-300 (1993).

[11] D. König, "Gráfok és mátrixok", Matematikai és Fizikai Lapok 38, 116-119 (1931), [in Hungarian].

[12] M. Kubale, "Interval vertex-coloring of a graph with forbidden colors", Disc. Math. 74, 125-136 (1989). 